ANNALES

POLONICI MATHEMATICI

LVII.3 (1992)

\title{
Most random walks on nilpotent groups are mixing
}

\author{
by R. RĘBOWSKI (Wrocław)
}

Abstract. Let $G$ be a second countable locally compact nilpotent group. It is shown that for every norm completely mixing (n.c.m.) random walk $\mu, \alpha \mu+(1-\alpha) \nu$ is n.c.m. for $0<\alpha \leq 1, \nu \in P(G)$. In particular, a generic stochastic convolution operator on $G$ is n.c.m.

1. Introduction. Let $G$ be a locally compact group with a left Haar measure $m$. We denote by $M(G)$ the convolution Banach algebra of finite Radon measures on $G$. The convex closed subset of (Radon) probabilities on $G$ will be denoted by $P(G)$. For a Banach space $X, \mathcal{L}(X)$ denotes the Banach algebra of bounded linear operators on $X$. An operator $T \in \mathcal{L}(M(G))$ is called a convolution operator if for some $\mu \in M(G)$ it is equal to $T_{\mu}$ or ${ }_{\mu} T$, where $T_{\mu} \nu=\nu * \mu$ and ${ }_{\mu} T \nu=\mu * \nu, \nu \in M(G)$. Thus the mapping $\mu \rightarrow T_{\mu}$ $\left({ }_{\mu} T\right)$ is a representation of $M(G)$ by a semigroup of right (left) convolution operators on $M(G)$. We let $L^{1}(m)$ be the Banach space of real-valued $m$ integrable functions on $G$. Then for each $\mu \in P(G), T_{\mu}$ and ${ }_{\mu} T$ are stochastic operators on $L^{1}(m)$, i.e. they take $P(G) \cap L^{1}(m)$ into itself.

It is well known that there is a 1-1 correspondence between the stochastic convolution operators on $L^{1}(m)$ and the random walks on $G$. This means that for a given random walk with law $\mu \in P(G)$, the right transition probability $p_{\mu}(g, \cdot)=\delta_{g} * \mu$ defines a stochastic convolution operator $T_{\mu}(\nu)=\int p_{\mu}(g, \cdot) d \nu(g), \nu \in M(G)$. We say that $T_{\mu}$ is induced by the random walk $\mu$. Analogously ${ }_{\mu} T$ is induced by the left transition probability ${ }_{\mu} p$.

Consider a right random walk with law $\mu$. A bounded Borel function $f$ on $G$ is called $\mu$-harmonic if it is $p_{\mu}$-invariant, or equivalently, if $\int f(g h) d \mu(h)=$ $f(g)$ for every $g \in G$ (for the left random walk ${ }_{\mu} p$ the definition is similar). If all the $\mu$-harmonic functions are constant, we say that the ran-

1991 Mathematics Subject Classification: 47A35, 22D40, $60 \mathrm{~J} 10$.

Key words and phrases: stochastic operator, convolution operator, random walk, norm completely mixing, nilpotent group. 
dom walk is ergodic. It is easy to check that the random walk is ergodic iff the induced stochastic convolution operator is ergodic, i.e. constant functions are the only fixed points for the adjoint to $T_{\mu}$ (resp. to ${ }_{\mu} T$ ) acting on $L^{\infty}(m)$.

We say that a random walk is norm completely mixing (n.c.m.) if the corresponding stochastic convolution operator is norm completely mixing, i.e. if for every pair $\nu_{1}, \nu_{2} \in P(G) \cap L^{1}(m)$ we have $\lim \left\|T_{\mu}^{n}\left(\nu_{1}-\nu_{2}\right)\right\|=0$ (resp. for ${ }_{\mu} T$ ).

Rosenblatt proved that there exists at least one n.c.m. random walk iff $G$ is $\sigma$-compact and amenable ([9], Thm. 1.10 and Prop.). As was observed in [6], if $G$ is second countable and abelian, the set of n.c.m. random walks forms a dense $G_{\delta}$ subset of the set of all stochastic convolution operators on $L^{1}(m)$ for both strong operator topology (s.o.t.) and norm operator topology (n.o.t.) ([6], Thm. 5).

We shall extend the above result to second countable nilpotent groups.

2. Generic stochastic convolution operator on a nilpotent group is n.c.m. Let $G$ be a second countable l.c. group with a left Haar measure $m$. The proof of Thm. 5 in [6] was based on the classical Choquet-Deny theorem, which says that in abelian groups, a random walk $\mu$ is ergodic iff the closed subgroup generated by the support of $\mu$ is the whole group $G$ (see e.g. [8], Ch. 5). Unfortunately, this is not true in general l.c. groups. As follows from the theory of Poisson spaces, for some random walks on nilpotent groups the Choquet-Deny theorem does hold (see [1], Prop. IV.10). For the reader's convenience we present some of the relevant facts of this theory. For the proofs see [1].

Take a random walk with law $\mu \in P(G)$. Then the space of all uniformly continuous $\mu$-harmonic functions is isometrically isomorphic to a certain $C^{*}$ commutative algebra with unit. Its spectrum $\Pi_{\mu}$, which is a compact $G$-space, is called the Poisson space of $G$ corresponding to the random walk $\mu$. The Gelfand transform of this algebra is called the Poisson formula. The Poisson formula determines all $\mu$-harmonic functions if $\mu$ is spread-out, i.e. $\mu^{* n}$ is not singular with respect to $m$ for some positive integer $n$ ([1], Thm. I.3).

Now assume $G$ is amenable. By combining Thm. I.3, Prop. IV.7 and Prop. IV.8 of [1], the following conditions are seen to be equivalent for a (right) random walk with a spread-out law $\mu$ :

(i) The space of $\mu$-harmonic functions is finite-dimensional;

(ii) $\Pi_{\mu}$ is finite;

(iii) $\Pi_{\mu}$ is isomorphic to $G / H$ as a $G$-space, where $H$ is the closed subgroup generated by the support of $\mu$; 
(iv) $\Pi_{\mu}$ is a homogeneous $G$-space.

Now, we see that the stochastic convolution operator $T_{\mu}$ is ergodic iff $G=H$ and $\Pi_{\mu}$ is homogeneous. Since in the case of nilpotent groups and $\mu$ spread-out, $\Pi_{\mu}$ is homogeneous iff $H$ has a finite index in $G$ ([1], Prop. IV.10), the above remark shows that for nilpotent groups with spreadout measures the classical Choquet-Deny theorem holds.

THEOREM. Let $G$ be a second countable nilpotent l.c. group. For every n.c.m. random walk with spread-out law $\mu$ the random walk $\mu_{\alpha}=\alpha \mu+$ $(1-\alpha) \nu$ is n.c.m. for every $\nu \in P(G)$ and $\alpha \in(0,1]$.

P r o of. From $\S 5$, Ch. 2 of [5], the assumption that $\mu$ is spread-out and n.c.m. is equivalent to $\left\|\left(\nu_{1}-\nu_{2}\right) * \mu^{* n}\right\| \rightarrow 0\left(\nu_{1}, \nu_{2} \in P(G)\right)$. This means that the random walk induces an ergodic "space-time" random walk on $G \times \mathbb{Z}$, where $\mathbb{Z}$ is the group of integers (see Lemma 3 of [7]). Now it is clear that the support of $\mu$ is not contained in a coset of a proper closed subgroup of $G$. Therefore, the same holds for the support of $\mu_{\alpha}$. To complete the proof apply the Choquet-Deny theorem and Thm. 2 of [3].

It is worth pointing out that the above theorem also follows from Proposition 2.5 of [9] and from the fact that for nilpotent groups the Choquet-Deny theorem holds.

COROLlaRY 1. The set of n.c.m. random walks on a nilpotent group $G$ is dense in the norm topology of $P(G)$.

Proof. We only need to show that on a nilpotent group there is at least one n.c.m. random walk with a spread-out law. Since nilpotent groups are amenable, this follows from the Rosenblatt theorem [9] (alternatively we can use the Choquet-Deny theorem).

CoROLlary 2. If $G$ is nilpotent, then the set of n.c.m. stochastic convolution operators is a dense $G_{\delta}$ set in the set of all stochastic convolution operators for both s.o.t. and n.o.t.

Proof. First note that the representation $\mu \rightarrow T_{\mu}\left({ }_{\mu} T\right)$ is norm continuous. Now the set of n.c.m. stochastic convolution operators, being the intersection of the sets of n.c.m. stochastic operators and the stochastic convolution operators, is a $G_{\delta}$ in s.o.t. (see Thm. 3 of [6] and the Wendel Theorem of [4]). Therefore, Corollary 2 follows from Corollary 1.

Recently, the author was informed by W. Bartoszek that Corollary 1 holds for arbitrary amenable $\sigma$-compact l.c. groups [2]. It is not known whether our Theorem is also true in that case. 


\section{References}

[1] R. Azencott, Espaces de Poisson des groupes localement compacts, Lecture Notes in Math. 148, Springer, Berlin 1970.

[2] W. Bartoszek, On the residuality of mixing by convolution probabilities, preprint.

[3] S. Glasner, Qn Choquet-Deny measures, Ann. Inst. Henri Poincaré 12 (1976), 1-10.

[4] E. Hewitt and K. Ross, Abstract Harmonic Analysis, Vol. 2, Springer, Berlin 1970.

[5] H. Heyer, Probability Measures on Locally Compact Groups, Springer, Berlin 1977.

[6] A. I wanik and R. Rębowski, Structure of mixing and category of complete mixing for stochastic operators, Ann. Polon. Math. 56 (1992), 233-242.

[7] B. Jamison and S. Orey, Markov chains recurrent in the sense of Harris, Z. Wahrsch. Verw. Gebiete 8 (1967), 41-48.

[8] D. Revuz, Markov Chains, North-Holland Math. Library, 1975.

[9] J. Rosenblatt, Ergodic and mixing random walks on locally compact groups, Math. Ann. 257 (1981), 31-42.

INSTITUTE OF MATHEMATICS

TECHNICAL UNIVERSITY OF WROCEAW

WYBRZEŻE WYSPIAŃSKIEGO 27

50-370 WROCŁAW, POLAND

Reçu par la Rédaction le 15.11.1991

Révisé le 20.5.1992 